\title{
EL PROCESO DE DESARTICULACIÓN DE LAS BASES ECONÓMICAS TRADICIONALES Y SUS POSIBLES ALTERNATIVAS EN LOS ANCARES LEONESES
}

\author{
José Ignacio GONZÁLEZ RAMOS y Joaquín GONZÁLEZ VECÍN
}

\section{INTRODUCCIÓN}

En consultas bibliográficas realizadas sobre libros geográficos de épocas pasadas, tanto de autores españoles como extranjeros, la provincia de León aparece como un espacio con grandes diferencias físicas y humanas; ello se debe al hecho de existir un medio de montaña que abarca todo el norte y gran parte del oeste de la provincia y un espacio predominantemente llano -la Meseta-, pero también porque en cada uno de estos grandes conjuntos se dan diferencias apreciables ya sea entre las vegas y riberas, frente a los secanos de la Maragatería o de Tierra de Campos, en el caso de la Meseta, o entre las imponentes moles montañosas de los Picos de Europa y las montañas en proceso de «disección» de la parte occidental de la provincia.

En el plano humano, estas diferencias se reiteran al describir modas y costumbres de las distintas comarcas de uno y otro medio. Así, alguno de esos autores, con aficiones que podríamos denominar geoetnográficas hace hincapié en las diferencias en la mentalidad, en las modas y, sobre todo, en el lenguaje, señalando que, dentro de la misma provincia, los habitantes de uno y otro extremo apenas se entienden entre sí.

Reduciendo el ámbito de estudio a la comarca del Bierzo, estas diferencias físicas y humanas resultan también sorprendentes si comparamos la hoya berciana con el anillo montañoso que la rodea; también se pueden apreciar entre la montaña occidental y la oriental e incluso, dentro de la hoya entre el Alto y el Bajo Bierzo.

Algunos geógrafos de este siglo han llamado la atención sobre la personalidad de los distintos valles existentes en el anillo montañoso, que implican una serie de matices comarcales dentro de la unidad general de toda la comarca.

Algunos de ellos - Valle de Fornela, Valle de Ancares, Valle de Valdueza, Valle de Boeza, etc.- aparecen diferenciados como comarcas no sólo en autores ya clásicos, sino también en publicaciones más recientes como el

\footnotetext{
${ }^{1}$ Entre otros: TERÁN Álvarez (1952-56) y OTERo PEDRAYo (1926),
} 


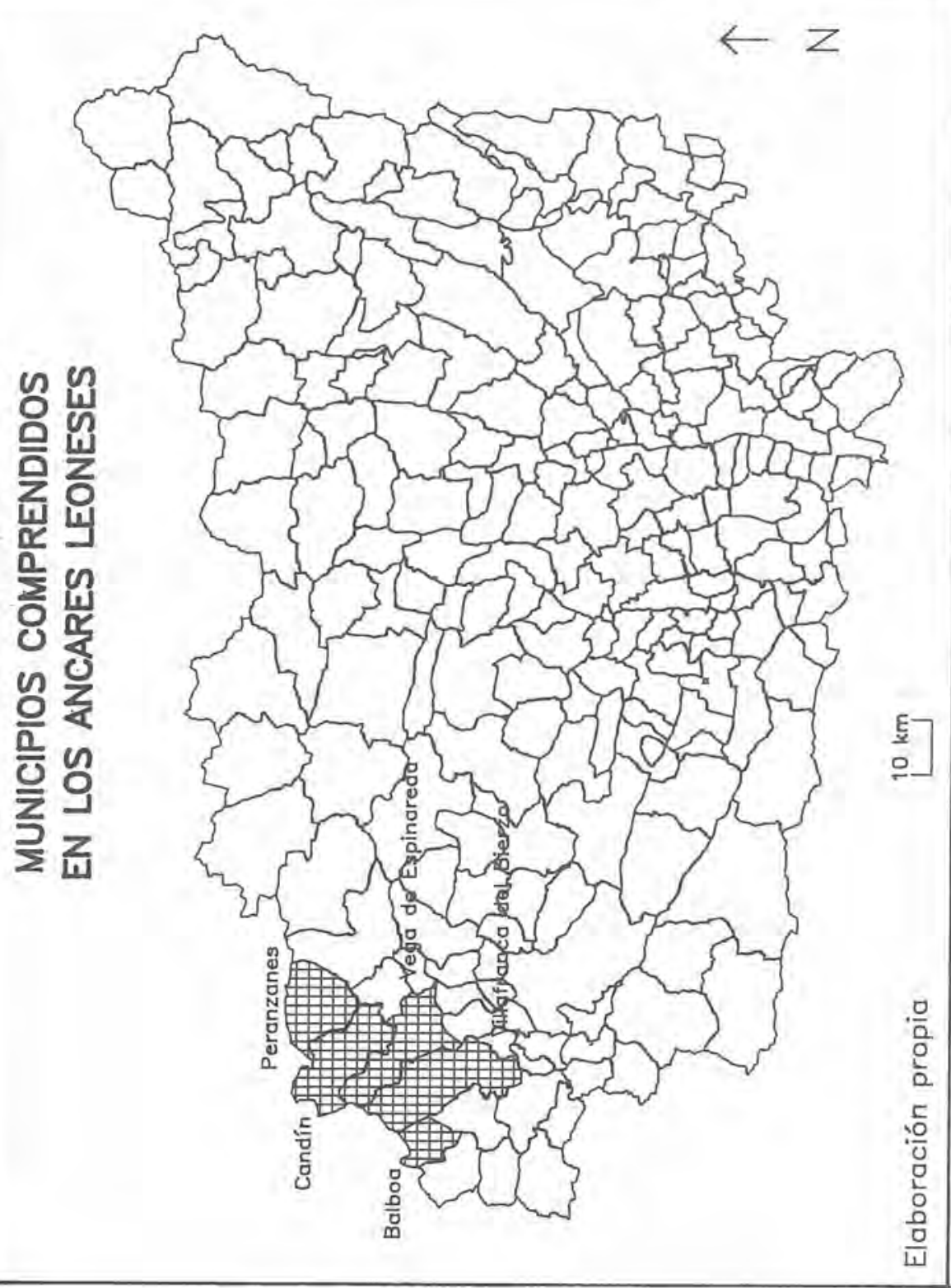




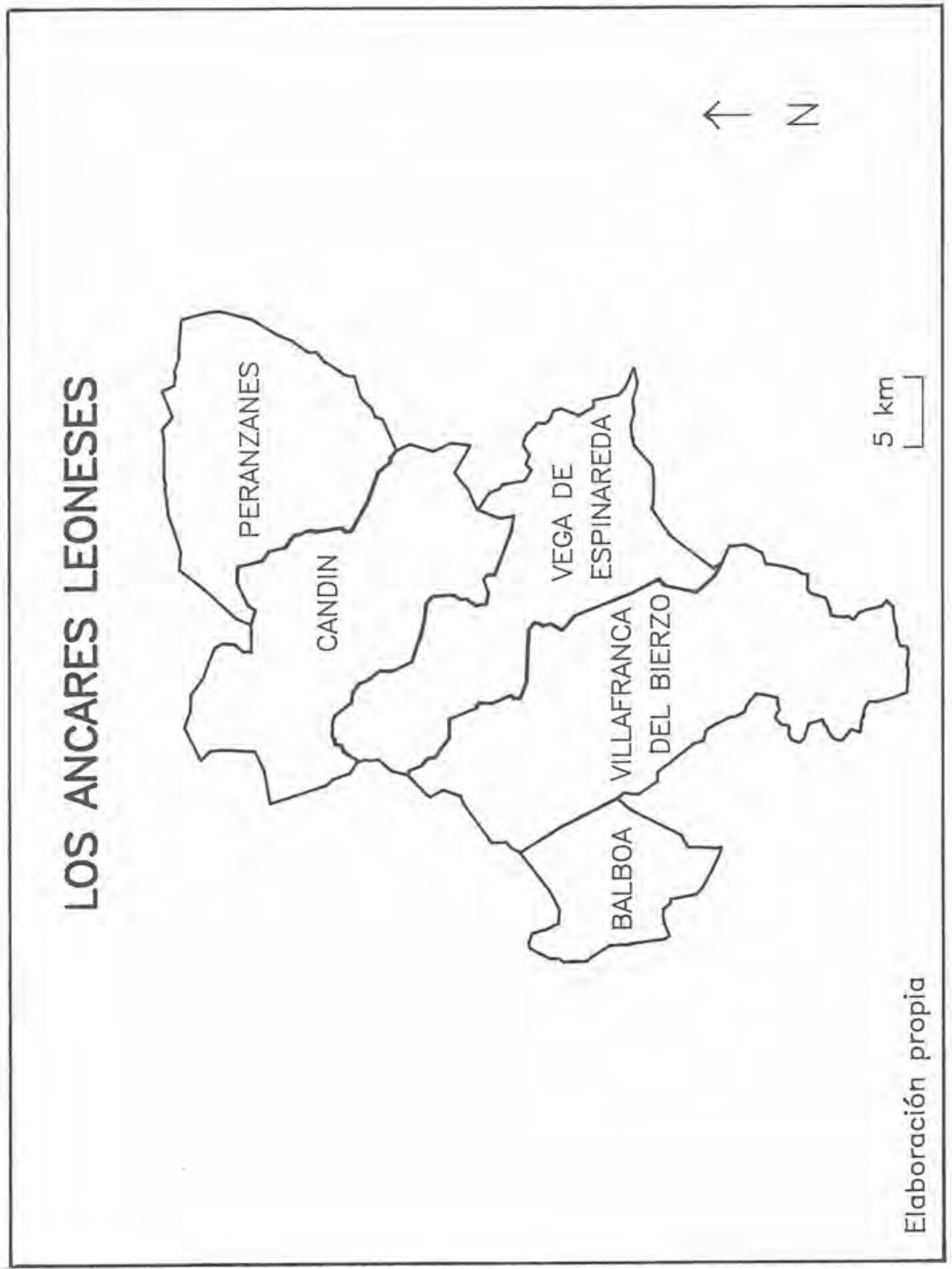


Mapa Provincial del Instituto Geográfico Nacional del año 1984, en el cual aparecen esos valles perfectamente diferenciados como pequeñas comarcas. La utilización del término Ancares intentado delimitar el área objeto de estudio puede resultar problemática. De hecho, no está muy distante en el tiempo cierta polémica, surgida por la realización de diversos proyectos (Cultural Campo-Sierra de Ancares, Proyecto Ancares) en los que la división territorial tradicional que circunscribía el término Ancares al valle avenado por este río y sus afluentes además de los pueblos de Suárbol y Balouta -prolongándose en la provincia de Lugo- ha sido tergiversada y el nombre de Sierra de Ancares ha adquirido categoría de comarca y ha comprendido todo el territorio comprendido en ésta, aunque aparecen subsistemas montañosos como la Sierra del Padrón y la Sierra de Mingatón englobados dentro de los Ancares. Este desconcierto se acentúa si tenemos en cuenta la delimitación de la Reserva Nacional de los Ancares Leoneses.

Superando las matizaciones que pueden surgir para delimitar la comarca de los Ancares y por razones de comodidad y mejor comprension, nosotros nos referimos con ese término al territorio comprendido en los términos municipales de Peranzanes, Candín y Balboa junto con parte del antiguo ayuntamiento de Valle de Finolledo y la totalidad del antiguo ayuntamiento de Paradaseca, respetando las particularidades que individualizan a cada uno de estos valles -Fornela, Ancares, La Somoza (Charrería)- pero partiendo del profundo convencimiento de que el proceso que pretendemos analizar reviste características muy similares en toda esta zona, por no hablar de otras similitudes de carácter cultural en un sentido amplio.

En el momento presente, estos ayuntamientos dan, en todos los indicadores socioeconómicos, los índices más bajos de la provincia junto con otros limítrofes, bien en la propia comarca o en otras como La Cabrera, e incluso en las provincias colindantes de Lugo y Oviedo; todos ellos englobados dentro de la extensa bolsa de pobreza, arcaísmo y marginación que constituye el noroeste montañoso peninsular, que presenta como una de sus características comunes la consideración de Parques o Reservas Nacionales como forma de acceder a ayudas estatales concretas.

A pesar de estas y otras ayudas, se puede afirmar con certeza que las mismas han sido insuficientes para crear una base de desarrollo socioeconomico de estos espacios, lo que no supone ignorar el reconocimiento de algunas mejoras, -construcción de vías de comunicación, instalación de teléfonos, traída de aguas, alcantarillado, restauración de elementos arquitectónicos típicos, etc-, fruto de subvenciones recibidas como consecuencia de la declaración de Comarcas de Acción Especial en áreas deprimidas de montaña, promovidas por los distintos organismos oficiales provinciales, regionales o estatales.

Esta visión dramática sobre estos espacios en los que las ya tradicionales diferencias campo-ciudad permanecen gravemente descompensadas, a pesar de los últimos avances reseñados, es una constante histórica reflejada en todas las fuentes consultadas desde el Catastro de la Ensenada de mediados del s. XVIII, los diccionarios geográficos de Miñano y Madoz de comienzos 
y mediados del XIX, libros geográficos de autores ya citados e incluso las descripciones sobre las condiciones de vida reflejadas en los Informes de los Mapas de la Comisaría de Abastecimientos y Transporte de la postguerra, hasta épocas más recientes donde siguen apareciendo como ejemplo de aislamiento o marginación.

En los últimos treinta años, esta zona ha sufrido un proceso de despoblación muy importante que, a nuestro juicio, está en el origen de lo que llamamos ruina de la explotación tradicional del espacio. Analizar este proceso y plantear las posibles alternativas son los dos objetivos que nos hemos planteado en este trabajo.

\section{EL PROCESO DE DESARTICULACIÓN DE LAS BASES ECONÓMICAS TRADICIONALES}

\subsection{Demografía: Despoblación y Envejecimiento}

Sobre los dos cuadros siguientes se podrían sacar numerosas conclusiones. Para los fines que nos proponemos destacaríamos las siguientes:

- A Io largo del siglo XX se observa, salvo en breves períodos intercensales, una pérdida de población que supera prácticamente en todos los casos el $50 \%$, pero que en algunos municipios se aproxima al $80 \%$. Se podría establecer como primera conclusión que una de las características demográficas de esta zona a lo largo de este siglo es la disminución de su población. Esa disminución se acentúa a partir de los años sesenta.

- La causa fundamental viene dada por los distintos procesos migratorios que han tenido desigual intensidad en el tiempo y en el espacio. Se puede decir que los municipios de esta zona han participado en todos estos procesos: emigración americana, europea y migraciones interiores de carácter nacional, provincial y comarcal. En la base de los mismos se encuentra la relativa «superpoblación» existente en ciertos períodos, superpoblación que no se deduce de la densidad demográfica -muy baja-, sino en la poca capacidad de la zona para generar recursos económicos.

- En la actualidad, la disminucíon de la población se ve influenciada no sólo por procesos emigratorios -que han perdido fuerza-, sino también por el propio crecimiento vegetativo negativo, fruto de una estructura poblacional por edades totalmente descompensada en la que la población joven es muy poco representativa, llegándose a una situación de incapacidad biológica de renovación. Este último aspecto constituye, a nuestro juicio, un problema fundamental y que lo diferencia de períodos anteriores: la pérdida de elementos jóvenes ha sido tan intensa que en los próximos años el proceso de despoblación se acentuará.

- Los cuatro municipios analizados forman parte de un espacio marginal en proceso de desertización desde el punto de vista demográfico. Para llegar a esta conclusión hay que tener en cuenta, además de las bajas densidades de población, la existencia de entidades locales próximas al despoblamiento: Fuente de Oliva, Parajís, Ruideferros y Ruidelamas, en el Municipio de 
Balboa; Suárbol en el de Candín; en este último Municipio el despoblamiento ya es efectivo en Villarbón.

- Si pasamos del ámbito municipal al análisis pormenorizado de las entidades locales menores, el cuadro resulta desolador y reafirma los niveles de desertización demográfica y la incapacidad biológica de renovación a la que hemos hecho referencia, Así, en el municipio de Balboa, las entidades de Chandevillar y Cantejeira no presentan ningún individuo entre 0 y 9 años y en el de Candín ocurre lo mismo, pero agravado: en Espinareda de Ancares y en Lumeras no hay individuos en los grupos de 0 a 14 años; en Suárbol de 0 a 59 y en Villasumil de 0 a 19; en el de Peranzanes en Chano, Cariseda y Fresnedelo no hay individuos de 0 a 4 años, en Faro de 0 a 9 y en Guímara de 0 a 14 hay tres individuos.

En estas valoraciones generales cabrían ciertas matizaciones relativas, sobre todo, a la diferencias entre ciertas cabeceras de municipio y entidades menores de esos mismos municipios o entre ciertos pueblos de valle y otros de montaña, pero, a nuestro juicio la conclusión clara que podemos establecer es que la dinámica demografica de los últimos treinta años se encuentra en la base de lo que se puede denominar "proceso de desarticulación del modo de vida tradicional de los Ancares $»^{2}$ ya que se está llegando, o ya se ha llegado en algunas entidades locales menores, a una situación en la que, no

Cuadro n. ${ }^{\circ} 1$

Evolución de la población de hecho a nivel municipal a lo largo del siglo

\begin{tabular}{|r|r|r|r|r|}
\hline & BALBOA & CANDÍN & PARADASECA & PERANZANES \\
\hline 1900 & 1.276 & 2.188 & 2.208 & 1.702 \\
\hline 1910 & 1.527 & 2.132 & 2.206 & 1.651 \\
\hline 1920 & 1.509 & 1.886 & 2.206 & 1.730 \\
\hline 1930 & 1.402 & 1.845 & 2.414 & 1.597 \\
\hline 1940 & 1.393 & 1.823 & 2.331 & 1.758 \\
\hline 1950 & 1.312 & 1.932 & 2.470 & 1.719 \\
\hline 1960 & 1.158 & 1.520 & 2.102 & 1.512 \\
\hline 1970 & 1.147 & 912 & 1.767 & 1.253 \\
\hline 1975 & 1.048 & 746 & -3 & 811 \\
\hline 1981 & 809 & 495 & 867 & 454 \\
\hline 1986 & 611 & 609 & 929 & 381 \\
\hline
\end{tabular}

\footnotetext{
${ }^{3}$ No disponemos de datos para ese año.
} VECÍN

2 Grupo de alumnos de Geografía Agraria bajo la dirección de Joaquín GONZÁLEZ 
Cuadro n. ${ }^{\circ} 2$

Evolución de la población. Variación porcentual intercensal

\begin{tabular}{|l|c|c|c|c|}
\hline & BALBOA & CANDÍN & PARADASECA & PERANZANES \\
\hline $1900-10$ & 19,2 & $-2,5$ & $-0,1$ & $-3,0$ \\
\hline $1910-20$ & $-0,8$ & $-11,5$ & 0,0 & 4,7 \\
\hline $1920-30$ & $-7,09$ & $-2,1$ & 9,4 & $-7,7$ \\
\hline $1930-40$ & $-0,6$ & $-2,1$ & $-3,5$ & 10 \\
\hline $1940-50$ & $-5,8$ & 5,9 & 6 & $-2,3$ \\
\hline $1950-60$ & $-11,7$ & $-21,3$ & -15 & -13 \\
\hline $1960-70$ & $-0,9$ & -40 & $-15,9$ & $-17,2$ \\
\hline $1970-75$ & $-8,6$ & $-18,2$ & $--^{3}$ & $-35,3$ \\
\hline $1975-81$ & $-22,8$ & $-33,6$ & $-50,9^{4}$ & $-45,01$ \\
\hline $1981-86$ & $-18,4$ & $-3,03$ & 7,15 & 6,5 \\
\hline
\end{tabular}

Fuente: Censos y Padrones de Población del I.N.E. Elaboración propia.

\section{Cuadro n..$^{2} 3$}

Población menor de cuarenta años en porcentajes sobre la población de hecho. Año 1986

\begin{tabular}{|l|r|}
\hline BALBOA & 36,70 \\
\hline CANDÍN & 27,08 \\
\hline PARADASECA & 41,60 \\
\hline PERANZANES & 39,15 \\
\hline
\end{tabular}

Fuente: Padrón Municipal de Habitantes de 1986.

Elaboración propia.

no sólo ha disminuido la capacidad de la poblacion para generar individuos que potencialmente puedan emigrar, sino que pone en duda la propia renovación biologica.

\subsection{Recursos Agrícolas: Usos del suelo y regímenes de tenencia de la tierra}

El aprovechamiento del suelo es fundamentalmente de carácter agrícola-ganadero. Al encontrarnos en un medio de montaña, se puede decir que el medio natural determina una aptitud más conveniente para la producción ganadera y el aprovechamiento forestal. Sin embargo, históricamente, las estructuras sociales han determinado una actividad predominantemente agrtcola (AgRicultuRA DE AUTOCONSUMO) a la que servía como complemento

\footnotetext{
${ }^{4}$ Dato referido al períoda $1970-81$.
} 
indispensable una ganadería poco seleccionada que, a la vez que producía estiércol, constituía un elemento fundamental de trabajo, alimentación y comercialización, prevaleciendo unas u otras funciones en relacion con el tipo de ganado. Se trataba, en definitiva, de subsistir en un medio que históricamente ha presentado cierto grado de aislamiento, pero donde, además, las necesidades de alimentación primaban sobre cualquier posibilidad de comercialización de los productos.

Todo ello se plasma en una organización-tipo del espacio cultivado que se repite prácticamente en todos los núcleos de población:

- Terrazgo dedicado a hortalizas, próximo al núcleo de población e incluso dentro del mismo. Ocasionalmente, se ha utilizado para cereales (cebada y, en contadas ocasiones, trigo) o para maíz (forraje). Puede tratarse de regadío o de secano.

- Praderío, bien aprovechando los valles o las laderas montañosas («lameiras/os»); en este último caso, el riego se hace de forma casi artesanal, por medio de pozos construidos en superficie.

- Terrazgo dedicado a cereales, fundamentalmente centeno, en régimen de barbecho o ganando terreno al monte mediante rozas periodicas de retama y posterior quema («searas»); ya en el Catastro de Ensenada se distingue entre tierras centenales que producen cada dos años y las que producen cada diez.

- Terrazgo de aprovechamiento arboreo bien formando «sotos» (agrupados) o aislados en el caso de castaños, nogales o ciertos árboles frutales; además existe una importante vegetación ripícola.

A continuacion se encontrarían los «montes comunales» objeto de aprovechamiento bien como combustible (roble, raíces de brezo -«garrochas»o cepas-, etc.), bien para alimento del ganado que alcanzaría su máxíma expresión en el aprovechamiento de brañas a partir de cierta altitud.

La única excepción en la zona estudiada vendría dada por el terrazgo dedicado a viñas en el antiguo término municipal de Paradaseca, fundamentalmente en torno a este núcleo de poblacion, aspecto que, en absoluto, puede considerarse significativo.

Si pasamos de estos aspectos meramente descriptivos a un análisis más profundo, utilizando diversos tipos de fuentes, nos interesa destacar las siguientes conclusiones:

- En cuanto al aprovechamiento del espacio llama poderosamente la atención el predominio absoluto del terreno improductivo o mínimamente productivo, al menos a efectos reales, consecuencia de la pobreza de los suelos y de la propia configuración del medio físico; en definitiva de la hostilidad del medio natural.

Este predominio ha variado a lo largo del siglo en funcion de factores demográficos, pero siempre ha presentado un porcentaje abrumador.

Los datos del cuadro n. ${ }^{9} 4$ son todavía más explícitos si utilizamos el Catastro de Rústica y se advierte el peso específico que tienen los terrenos agrupados bajo el epígrafe Monte de Utilidad Pública (M.U.P.).

En los datos del cuadro n..$^{2} 5$ no hemos incluido, en ningún caso, las propiedades comunales que, pese a tener un tratamiento fiscal diferente a los 
M.U.P., en la práctica, en la mayoría de los casos, su aprovechamiento es similar. A todo ello debemos añadir la reducción en el espacio cultivado, punto que trataremos posteriormente.

Cuadro n. 94

Superficie total de las explotaciones censadas en Has, y porcentualmente

\begin{tabular}{|c|c|c|c|c|c|c|c|c|c|}
\hline & Total & $\begin{array}{l}\text { Tierras } \\
\text { Labradas }\end{array}$ & $\%$ & $\begin{array}{l}\text { Prados Prad. } \\
\text { Permanentes } \\
\text { Pastizales }\end{array}$ & $\$$ & $\begin{array}{l}\text { Esp. Arbó- } \\
\text { reos Fores- } \\
\text { tales }\end{array}$ & $\%$ & Otras & $\%$ \\
\hline BALBOA & 5.181 & 294 & 56 & 268 & $5 ' 17$ & 566 & 10,9 & 4.054 & $78 / 2$ \\
\hline CANDÍN & 17.875 & 287 & 16 & 594 & $3{ }^{\prime} 32$ & 2546 & 14,2 & 14.447 & $80^{\prime} 8$ \\
\hline PARADASECA ${ }^{5}$ & $\cdots$ & $\ldots$ & $\ldots$ & $\ldots$ & $\ldots$ & $\cdots$ & $\cdots$ & -.. & ... \\
\hline PERANZANES & 11.033 & 29 & 0,26 & 42 & 0,38 & 1.405 & 12,7 & 9.558 & $86^{\prime} 6$ \\
\hline
\end{tabular}

Fuente: Censo Agrario 1,982. Elaboración propia.

Cuadro n. ${ }^{2}$ 5. Superficie término municipal $y$ superficie de M.U.P en Has. $y$ porcentualmente

\begin{tabular}{|l|r|r|c|}
\hline & Sup. término munic. & M.U.P. & $\%$ \\
\hline BALBOA & 5.209 & 3.086 & 59 \\
\hline CANDÍN & 14.687 & 12.837 & 87 \\
\hline PARADASECA & 13.857 & 9.832 & 71 \\
\hline PERANZANES & 11.019 & 10.572 & 96 \\
\hline
\end{tabular}

Fuente: Catastro de Rústica. Elaboración propia. ${ }^{6}$

- Un segundo aspecto a destacar, en cuanto a los usos del suelo, es la pervivencia de unas estructuras tradicionales de caracter secular.

Utilizando el Catastro de Rústica actual y comparándolo con los datos del cuadro n. ${ }^{2} 6$, se puede afirmar que las variaciones más importantes que se han producido en más de dos siglos vienen dadas por la desaparición del aprovechamiento de tierras dedicadas a lino y la introduccion de especies como la patata -de gran importancia en la alimentación humana y animal- $y$, en menor medida, el maíz.

Evidentemente, el cuadro $n .{ }^{9} 7$ no es comparable con el elaborado para el siglo XVIII, ya que aquel se refiere a cada entidad local y éste a la totalidad del municipio. Además hemos prescindido de algunos aprovechamientos que distorsionarían la realidad como el porcentaje ocupado por los Pastos?

\footnotetext{
${ }^{5}$ Los datos relativos a Paradaseca se incluyen en el municipio de Villafranca, por lo que para nuestro fin no son válidos.

${ }^{6}$ Para los términos municipales de Candín y Peranzanes se han utilizado las Respuestas Generales; para Balboa y paradaseca el Libro de Cédulas de 1.988 ya que las Respuestas Generales se estaban informatizando en aquellos momentos.

${ }^{7}$ La cantidad de Has. de terreno dedicado a Pastos es tal en todos los municipios analizandos que nos hace sospechar que en ellas se han incluido numerosos montes de aprovecha-
} 
Cuadro n. ${ }^{0}$ 6. Usos del suelo en el s. XVIII. Candín, Balouta, Balboa y Peranzanes (\% sobre el total)

\begin{tabular}{|c|c|c|c|c|c|c|c|}
\hline \multirow[t]{2}{*}{ CANDIN } & $\begin{array}{c}\text { Huertos } \\
\text { botralizas }\end{array}$ & $\begin{array}{c}\text { Tierras lina- } \\
\text { res }\end{array}$ & $\begin{array}{l}\text { Tierras cen- } \\
\text { tenales }\end{array}$ & $\begin{array}{c}\text { Prados rega- } \\
\text { dio }\end{array}$ & Castafios & Cerezo & Incul. \\
\hline & 0,11 & 0,78 & 40,47 & 9,94 & 3,76 & 0,04 & 44,79 \\
\hline \multirow[t]{2}{*}{ BALOUTA } & Huertos & $\begin{array}{l}\text { Tierras cen- } \\
\text { tenales }\end{array}$ & $\begin{array}{l}\text { Prados rega- } \\
\text { dío }\end{array}$ & $\begin{array}{c}\begin{array}{c}\text { Prados secta- } \\
\text { no }\end{array} \\
\end{array}$ & Montes & & \\
\hline & 0,92 & 9,62 & 7,64 & 4,37 & 75,13 & & \\
\hline \multirow[t]{4}{*}{ BALBOA } & $\begin{array}{l}\text { Huertos } \\
\text { regadio }\end{array}$ & $\begin{array}{l}\text { Huertos } \\
\text { secano }\end{array}$ & $\begin{array}{l}\text { Linares } \\
\text { regadio }\end{array}$ & Linar secano & $\begin{array}{c}\text { Trigal seca- } \\
\text { no }\end{array}$ & \multicolumn{2}{|c|}{$\begin{array}{c}\text { Tierra centenal desc. } \\
1 \text { año } \\
\end{array}$} \\
\hline & 0,22 & 0,33 & 1,07 & 0,75 & 3,10 & \multicolumn{2}{|c|}{30,06} \\
\hline & $\begin{array}{l}\text { Prado rega- } \\
\text { dfo }\end{array}$ & $\begin{array}{c}\text { Prado seca- } \\
\text { no }\end{array}$ & Castaños & Inculta & $\begin{array}{l}\text { Caminos y } \\
\text { ríos }\end{array}$ & \multicolumn{2}{|c|}{$\begin{array}{c}\text { Tierra centenal desc. } \\
9 \text { años }\end{array}$} \\
\hline & 15,41 & 1,45 & 6,40 & 31,17 & 6 & \multicolumn{2}{|c|}{2,10} \\
\hline \multirow[t]{2}{*}{ PERANZANES } & $\begin{array}{l}\text { Tierras hor- } \\
\text { taliza }\end{array}$ & Linares & Centenales & $\begin{array}{c}\text { Prados rega- } \\
\text { dio }\end{array}$ & $\begin{array}{c}\text { Prados seca- } \\
\text { no }\end{array}$ & \multicolumn{2}{|c|}{ Tierras en monte } \\
\hline & 0,30 & 1,6 & 43,7 & 14,2 & 1,2 & \multicolumn{2}{|c|}{38,8} \\
\hline
\end{tabular}

Fuente: Catastro de Ensenada. Elaboración propia.

Cuadro n. 97 Aprovechamiento actual del suelo (en \%)

\begin{tabular}{|l|r|r|r|r|}
\hline & BALBOA & CANDÍN & PERANZANES & \multicolumn{1}{c|}{ PARADASECA } \\
\hline Labor Reg. & 0,33 & 0,28 & 3,95 & 1,54 \\
\hline Prado Reg. & 13,2 & 9,34 & 21,4 & 14,35 \\
\hline Labor Sec. & 72,8 & 63,2 & 54,3 & 72,1 \\
\hline Prado Sec. & 1,33 & 27 & 19,7 & 9,8 \\
\hline Viña & -- & - & - & 2,06 \\
\hline
\end{tabular}

Fuente: Catastro de Rústica. Elaboración Propia. Los datos se refieren a la totalidad del municipio.

o el arbolado. No obstante creemos que puede resultar significativo para reafirmarnos en esa explotación casi secular del suelo a la que hemos hecho referencia.

- En tercer lugar, debemos destacar que en los últimos años, y, en clara relación con aspectos demográficos ya apuntados, se puede observar una drástica reducción del terreno cultivado dedicado a cereales -predominantemente centeno-, que todavía no se detecta en las estadísticas oficiales, pero sí en la realización del trabajo de campo. La reducción ha sido general, pero en algunas entidades locales su presencia, cuando se produce, es meramente anecdotica. Este terreno tiene un primer aprovechamiento de tipo ganadero («poulas») a finales de primavera y principios del verano. En muchos casos pasa a formar parte de las amplias zonas de matorral -retama y brezo- 0

miento comunal cuya utilización ganadera no es significativa. 
monte bajo que de forma peribdica es pasto de las llamas.

Aunque minoritario y todavía poco significativo, ya se puede detectar el abandono de algunos terrenos dedicados a pastos en las laderas montañosas («Lameiras») e incluso en algunos dedicados a hortalizas. En un futuro muy próximo, salvo circunstancias externas, este fenomeno se generalizará.

Todo ello hace que en la última década se produzca una disminución de la producción agrícola lo cual no implica como tendremos ocasión de demostrar una reorientación hacia el sector ganadero, sino que es fruto de la interrelación de diversos factores: envejecimiento progresivo de la población, generalización de las jubilaciones y, por tanto, desaparición de la necesidad perentoria de cultivar para consumir, posibilidad de acceso a producto alimenticios que se encuentran en el mercado, etc.

\section{Regímenes de tenencia de la tierra}

- Historicamente, el cambio más importante se produce a lo largo del siglo XIX, cuando se configura una propiedad privada de la tienra que sustituye a otras formas de propiedad características del Antiguo Régimen.

- En la actualidad, dos características sobresalen sobre cualesquiera otras:

(i) La exigüidad de las explotaciones.

(ii) La excesiva parcelación de las mismas, que le dan al conjunto de los cuatro municipios un carácter que nos atrevemos a llamar de «microfundio».

Cuadro n. ${ }^{2}$ 8. Tamaño de las explotaciones, porcentualmente, para los municipios estudiados

\begin{tabular}{|l|r|r|r|r|r|r|}
\hline & - de $1 / 2 \mathrm{Ha}$. & $1 / 2-1 \mathrm{Ha}$. & $1-3 \mathrm{Ha}$. & $3-5$ Has & $5-10 \mathrm{Has}$ & $+10 \mathrm{Has}$. \\
\hline BALBOA & 39,8 & 16 & 23,7 & 8 & 7,9 & 4,36 \\
\hline CANDIN & 40,4 & 15,03 & 23,09 & 10,2 & 7,3 & 1,85 \\
\hline PARADASECA & 55,7 & 14,6 & 19,55 & 6,54 & 2,48 & 1 \\
\hline PERANZANES & 61,96 & 21,34 & 13,9 & 1,1 & 0,36 & 1,22 \\
\hline
\end{tabular}

Fuente: Catastro de Rústica -Listado Alfabético. Elaboración propia.

A nuestro juicio, el cuadro es lo suficientemente significativo como para que las diferencias existentes entre los diversos municipios carezcan de relevancia.

Como factores agravantes de esta situación, si cabe, hay que señalar los siguientes:

- Han sido incluidos tanto los Montes de Utilidad Pública como las propiedades comunales. En algún caso -Peranzanes- este concepto supone la totalidad de las propiedades mayores de 10 Has. .

- En muchos casos, la realidad que nos ofrece el trabajo de campo es todavía más espeluznante: en el municipio de Balboa, un 18\% de los propietarios aparece con epígrafes que indican una mayor división real (Herederos, hermanos, y 1,2, etc.) y en este mismo municipio, los 24 propietarios parti- 
culares que figuran con una propiedad mayor de 10 Has. han fallecido, 10 que «a priori» puede suponer mayor división, aunque no necesariamente.

- De forma general, el numero de propietarios, lejos de disminuir, como cabría suponer, teniendo en cuenta la evolución demográfica, ha aumentado, sobre todo tras la desaparición de la «Manda»-especie de variante gallega del Mayorazgo- produciéndose el reparto equitativo de los bienes entre los distintos hermanos.

- Resulta significativo que el número de propietarios- contribuyentes supere, no ya al número de vecinos, sino al número de habitante hasta duplicarlo en muchos casos.

Cuadro n. ${ }^{\circ}$ 9. Habitantes (1986) y Propietarios/Contribuyentes (1988)

\begin{tabular}{|l|r|r|r|}
\hline & Habitantes (1986) & Propietarios & Contribuyentes \\
\hline BALBOA & 660 & 984 & 979 \\
\hline CANDÍN & 480 & 1.024 & 1.015 \\
\hline PARADASECA & 929 & 2.291 & 2.275 \\
\hline PERANZANES & 475 & 815 & 806 \\
\hline
\end{tabular}

Fuentes: Padrón Municipal de Población; Catastro de Rústica-Listado Alfabético y Catastro de Rústica -Libro de Cédulas 1.988.

Incluimos el número de contribuyentes porque el de propietarios incluye los M.U.P. y los bienes comunales y de Juntas Vecinales; a pesar de todo, los datos se comentan por sí sólos: el proceso de emigración y de disminución de la población no se ha plasmado en un proceso paralelo de concentración de la propiedad.

Como formas de explotación, es totalmente mayoritaria la explotación directa; en cuanto al arrendamiento, se produce un tipo de arrendamiento casi estructural sobre propiedades de forasteros y otro más reciente que, en ocasiones, sólo se detecta en el trabajo de campo y que no siempre implica una compensación económica pecuniaria por parte del arrendatario. Como norma general, no se dan formas contractuales: se trata de intercambios o acuerdos verbales.

Incluso exceptuando los Montes de Utilidad Pública, tiene cierta importancia la propiedad de carácter comunal en todos los municipios, pero sobre todo en Candín y Peranzanes.

Finalmente, señalar la existencia de ciertas propiedades que podemos denominar de carácter residual -propiedades del Obispado de Astorga. 
Cuadro n. ${ }^{2}$ 10. Régimen de Tenencia de la Tierra (en Has.)

\begin{tabular}{|l|r|r|r|r|}
\hline & Superficie & Propiedad & Arrendamiento & \multicolumn{1}{c|}{ Otras } \\
\hline BALBOA & 5.181 & 1.971 & 34 & 3.176 \\
\hline CANDÍN & 17.875 & 3.883 & 84 & 13.909 \\
\hline PERANZANES & 11.033 & 5.931 & 2 & 5.100 \\
\hline
\end{tabular}

\section{Cuadro n..$^{2} 11$}

Régimen de Tenencia sobre superficie agrícola utilizada (en Has.)

\begin{tabular}{|l|c|c|c|c|}
\hline & Superficie & Propiedad & Arrendamiento & Otras \\
\hline BALBOA & 562 & 541 & 20 & 1 \\
\hline CANDÍN & 882 & 548 & 82 & 252 \\
\hline PERANZANES & 71 & 69 & 2 & -- \\
\hline
\end{tabular}

Fuente: Censo Agrario 1982.

En cuanto a la excesiva parcelación, si exceptuamos los Montes de Utilidad Pública, la parcela-tipo del Municipio de Candín tendría una extensión aproximada de 13 Áreas y 64 Centiáreas y la del Municipio de Peranzanes sería de 7 Areas y 74 Centiáreas. En el primer caso, las parcelas de más de $1 \mathrm{Ha}$. representan el $0^{\prime} 43 \%$ del total, mientras que en el segundo son el $0^{\prime} 0-$ $9 \%$.

Consideramos que los aspectos tratados caracterizan y determinan la estructura económica de la zona que puede ser calificada como economía tradicional pero en claro proceso de descomposición, y a la vez, suponen un lastre cuya superación se nos antoja imposible al menos desde dentro de estas sociedades. Esta imposibilidad se comprende analizando el envejecimiento de la población lo que implica un desinterés por parte de los empresarios agrícolas de edades avanzadas.

Cuadro n. 12. Número de Empresarios-Personas Fisicas por grupos de edad y ocupacion principal.

\begin{tabular}{|l|r|r|r|r|r|r|r|r|}
\hline & Total & $\begin{array}{c}\text { Hasta } \\
34 \text { años }\end{array}$ & $35-54$ & $55-64$ & + de 65 & Agrar. & $\begin{array}{c}\text { No } \\
\text { agrar. }\end{array}$ & Otras \\
\hline BALBOA & 214 & 3 & 63 & 56 & 92 & 86 & 17 & 111 \\
\hline CANDfN & 194 & 3 & 46 & 68 & 77 & 138 & 6 & 50 \\
\hline PERANZANES & 231 & 2 & 70 & 67 & 92 & 30 & 27 & 174 \\
\hline
\end{tabular}

Fuente: Censo Agrario de 1982.

Teniendo en cuenta los aspectos anteriores, cualquier otra consideración (Escasa mecanización, pervivencia de labores realizadas con ganado de tiro, aperos de labranza arcaicos...etc.). no hace más que acentuar la visión de una zona en total proceso de desarticulación social y económica y que, por su intensidad, aparece como irreversible. 


\subsection{Recursos ganaderos}

Ya hemos señalado que las condiciones naturales de este medio de montaña posibilitaban unas mayores aptitudes para la explotación ganadera y forestal frente al aprovechamiento agrícola a pesar de la importancia que historicamente ha tenido éste.

Tradicionalmente, la ganadería ha constituido el otro recurso importante en la zona, sin que se pueda diferenciar de forma radical del aprovechamiento agrícola, pues ambos han sido claramente complementarios: difícilmente hubiese sido viable la agricultura de subsistencia sin el estiércol proporcionado por los animales y sin la utilización de su fuerza para el trabajo. Constituye también la fuente abastecedora de leche, carne, lana, etc., complementando así ese panorama cuya finalidad primordial era abastecer a los habitantes de esta zona de lo necesario para subsistir.

Por otra parte, la ganadería ha sido un sector enfocado, al menos en alguna de sus especies, a la comercializacion, frente a la agricultura cuyas posibilidades comerciales han sido ínfimas por diversos motivos, proporcionando así los ingresos pecuniarios necesarios para adquirir otros productos, pagar rentas, etc.; nos estamos refiriendo, sobre todo, al ganado ovino, caprino y bovino, ya que el porcino ha sido tradicionalmente de autoconsumo y los équidos han sido animales de carga y transporte.

La evolución reciente de la cabaña ganadera se puede observar en el siguiente cuadro:

Cuadro n.: 13. Número de cabezas de ganado en los años que se citan

\begin{tabular}{|l|r|r|r|r|r|r|}
\hline & Año & Bovino & Ovino & Caprino & Porcino & Equino \\
\hline \multirow{5}{*}{ BALBOA } & 1945 & 685 & 2.076 & 1.081 & 408 & 20 \\
\cline { 2 - 8 } & 1982 & 450 & 400 & 300 & 425 & 80 \\
\cline { 2 - 8 } & 1986 & 455 & 500 & 395 & 340 & 50 \\
\hline CANDIN & 1945 & 790 & 1.220 & 844 & 574 & 97 \\
\cline { 2 - 8 } & 1982 & 416 & 305 & 575 & 305 & 40 \\
\cline { 2 - 8 } & 1986 & 265 & 210 & 500 & 80 & 30 \\
\hline PARADASECA & 1945 & 1.510 & 2.800 & 2.820 & 690 & 120 \\
\cline { 2 - 8 } (Villafranca) & $1982^{8}$ & 1.019 & 860 & 1.054 & 873 & 193 \\
\cline { 2 - 8 } & $1986^{8}$ & 876 & 678 & 910 & 873 & 193 \\
\hline PERANZANES & 1945 & 415 & 414 & 916 & 141 & 31 \\
\cline { 2 - 8 } & 1982 & 107 & 38 & 513 & 35 & 9 \\
\cline { 2 - 7 } & 1986 & 123 & 107 & 342 & 84 & 5 \\
\hline
\end{tabular}

${ }^{8}$ Los datos se refieren a todo el municipio de Villafranca. No obstante, los incluimos porque pueden resultar muy significativos. 
Cuadro n." 14. Número de cabezas de ganado en los municipios de Balboa y antiguo de Paradaseca en el año $1988^{\circ}$

\begin{tabular}{|l|c|c|c|c|}
\hline & Bovino & Ovino & Caprino & Équidos \\
\hline BALBOA & 350 & 515 & 967 & 44 \\
\hline PARADASECA & 524 & 383 & 495 & 31 \\
\hline
\end{tabular}

En una primera aproximación, cabe señalar una disminucion general en el número de cabezas de ganado para todas las especies, pero especialmente acentuada en el ovino y caprino. Esta reducción coincide en el tiempo con la disminución de población, a la que ya hemos hecho referencia, y la reducción del espacio cultivado y se puede considerar un fenómeno paralelo y ligado a éstos.

\section{Cuadro n..$^{\circ} 15$}

Número de cabezas de ganado y de explotaciones ganaderas en los municipios de Balboa, Candin y Peranzanes en el año $1988^{10}$

\begin{tabular}{|c|c|c|c|c|c|}
\hline & & $\mathrm{N} .^{2}$ cabezas & $\begin{array}{l}\text { N. }{ }^{2} \text { Explota- } \\
\text { ciones }\end{array}$ & Explot. media & Total explot. \\
\hline \multirow[t]{3}{*}{ BALBOA } & Bovino & 404 & 106 & 3,8 & \multirow[b]{3}{*}{107} \\
\hline & Ovino & 514 & 12 & 42,8 & \\
\hline & Caprino & 428 & 6 & 71,3 & \\
\hline \multirow[t]{3}{*}{ CANDIN } & Bovino & 404 & 93 & 4,3 & \multirow[b]{3}{*}{96} \\
\hline & Ovino & 95 & 11 & 8,6 & \\
\hline & Caprino & 360 & 7 & 51,4 & \\
\hline \multirow[t]{3}{*}{ PERANZANES } & Bovino & 100 & 44 & 2,2 & \multirow[b]{3}{*}{55} \\
\hline & Ovino & 41 & 5 & 8,2 & \\
\hline & Caprino & 293 & 20 & 14,6 & \\
\hline
\end{tabular}

En los últimos ańos se puede observar cierta tendencia a la estabilización, al menos a falta de nuevos datos. A nuestro juicio, la tendencia general será a la disminución, salvo cierta estabilidad o incluso cierta recuperación en aquellas especies que más han disminuido, favorecidas por subvenciones de carácter oficial, siempre que no se produzcan cambios importantes en las formas de explotación y en la orientación económica. Incluso las sospechas

\footnotetext{
${ }^{9}$ Los datos de cuadro n. ${ }^{2} 13$ han sido tomados de Celada y Llamazares (1989).

${ }^{10}$ Los datos correspondientes al cuadro n. 914 nos han sido facilitados por la Sección de Estadística e Informática del Servicio Territorial de la Junta de Castilla y León. Ello puede explicar las diferencias en los datos manejados. De todas formas, sorprende el número de cabezas de bovino en Candín en el año 1988. El número total de explotaciones no coincide con la suma de las explotaciones porque existen propietarios que lo son a la vez de distintas especies,
} 
que puedan ofrecer ciertos datos estadísticos, pueden encontrar su explicación en el período en el que se realicen los inventarios. En el caso de Candín, de las 404 cabezas de ganado bovino debemos tener en cuenta que sólo 257 cabezas son cunsideradas «de ordeño» mientras que 147 son de «no ordeño» lo que nos pone en relación con aprovechamientos cárnicos incluso de tipo doméstico. En Peranzanes, entidad local, nos informaron que en el pueblo sólo había una decena escasa de cabezas de ganado bovino, mientras que en las brañas había al menos treinta, hasta Noviembre, en que eran conducidas al pueblo para ser sacrificadas en la matanza.

En cuanto a la explotación de los recursos ganaderos, a pesar de ciertas innovaciones, como la mejora en las razas bovinas, la caracteristica fundamental es la pervivencia de elementos tradicionales.

Analizando el cuadro n. ${ }^{2} 14$ llegamos a la conclusión de que se podría, hablar de un «minifundio» ganadero: solo hay que ver la media del número de cabezas por explotación. Pero es que en el conjunto de las explotaciones y en su aprovechamiento siguen presentes esas «coordenadas tradicionales»: en el bovino todavía es importantísima su aportación como animales de tiro, productores de leche para el consumo y «productores» de estiércol. La comercialización se realiza fundamentalmente en terneros que se engordan estabulados hasta los cinco o seis meses para venderlos en las ferias próximas; el ovino y el caprino se comercializa para carne y el porcino sigue siendo una especie destinada al autoconsumo (realización de la tradicional matanza).

La producción de leche sólo se ha comercializado en el valle de Balboa y en el último año se ha reducido drásticamente cuando la empresa que recogía la leche ha dejado de hacerlo.

Las pocas innovaciones que se pueden señalar vienen dadas por la mejora en las razas del ganado bovino (cruces con Rubia gallega o asturiana e introducción de la pardo-alpina, con la desaparición casi total de la llamada «vaca del país»), pues el ovino y el caprino no han conocido casi modificaciones $^{11}$. Se ha producido una mejora, aunque no generalizada, en las condiciones de los establos y, todavía en menor medida, en la alimentación, que se sigue basando en el pastoreo tradicional.

Otras especies ganaderas existentes no pasan de ser elementos destinados al autoconsumo -Aves- o intentos de poner en marcha explotaciones de tipo innovador que en muchos casos no han cristalizado -Conejos-; en este sentido, llama la atención la disminución de 500 conejas reproductoras en 1982 en Balboa a 100 en 1986. También existe una tradición apícola, pero el número de colmenas también tiende a disminuir y nos tememos que en próximos censos hayan desaparecido ${ }^{12}$.

La propiedad es, en su totalidad, directa, habiendo desaparecido formas tradicionales e históricas, fruto de la escasez de medios económicos, como

\footnotetext{
${ }^{11}$ Sobre el aspecto de las razas ganaderas Vid. Celada y Llamazares (1989).

${ }^{12}$ Datos tomados del Censo General Ganadero -1986.
} 
formas de aparcería denominadas en algunos de los municipios como «de cabana».

Podemos concluir señalando que los recursos ganaderos, tan importantes desde el punto de vista histórico ${ }^{13}$ no se sustraen, sin menospreciar ciertas innovaciones, a ese ambiente de explotación tradicional en proceso de desarticulación.

\subsection{Otros recursos complementarios}

Teniendo en cuenta el análisis económico que acabamos de hacer no puede sorprender la existencia de otras actividades complementarias que, en ocasiones se realizaban o realizan fuera de la zona.

Históricamente destaca la importancia de la arriería en el valle de Ancares y en el de Fornela, como ha sido señalado en numerosas ocasiones. S6́lo en la entidad local de Candín, el Catastro cita la existencia de 14 arrieros «trajinantes y comerciantes por mayor y menor en pescado fresco y seco, jabon, aceite, sardinas y otras especies», y en la entidad de Peranzanes «hay 39 arrieros» ${ }^{14}$.

En la actualidad, como herencia histórica de la arriería, destaca la venta ambulante en el valle de Fornela, aunque con crecientes dificultades debido a la prohibición para realizar este tipo de actividad por parte de algunos municipios o incluso, según opinión de los interesados, por crecientes trabas de tipo impositivo. Numerosos vendedores ambulantes se han establecido regentando sus propios comercios en núcleos de población proximos o incluso en otras provincias.

La producción artesanal ha sido en el pasado, un complemento indispensable y, a la vez, una actividad necesaria, para salir adelante en economías tan cerradas; esta producción se ha mantenido hasta tiempos relativamente recientes y abarcaba desde la elaboración de aperos de labranza, hasta calzado rudimentario (galochas), cestas, etc. En la actualidad, como tal actividad de carácter tradicional, prácticamente está en vías de desaparición.

En el municipio de Peranzanes destaca el porcentaje de población dedicada a actividades energéticas relacionadas con el mantenimiento del teleférico (baldes vaivén) que, atravesando longitudinalmente todo el valle, transporta el carbón desde la zona del Tormaleo en Asturias a las cercanías de Páramo del Sil. Esta actividad ocupa al $28 \%$ de la población activa de este municipio -aproximadamente unas 20 familias.

\footnotetext{
${ }^{13}$ Sobre la importancia histótica de la ganadería simplemente citar que según el Catastro, la comarca de la Somoza (antiguo munic. de Paradaseca) incluyendo a Burbia nos da 1811 cabezas de vacuno, 3.242 de Ovino, 4284 de Caprino, 597 de Porcino y 13 équidos, mientras que para el municipio de Balboa las cifras son 946, 2426, 2607, 805 y 10 según Celada y Llamazares (1989: 7-8). Nosostros mismos hemos constatado que la entidad de Balouta da unas cantidades de 163 de vacuno, 173 de Ovino, 132 de Caprino y 21 équidos, mientras que la de Candín nos da 44 de vacuno, 102 de Ovino, 92 de Cabrío y 27 de Equidos. Todo ello a pesar de posibles ocultaciones.

${ }^{14}$ Catastro del Marqués de Ia Ensenada. Archivo Histórico Provincial de León.
} 
Otros sectores que tienen una relativa importancia son la construccion, sobre todo en el antiguo municipio de Paradaseca y en el de Balboa en relación, bien con la reciente y creciente renovación del hábitat o con la realización de trabajos fuera de los municipios, y los desplazamientos diarios para trabajar en alguna industria de Ponferrada o en el sector servicios, sobre todo en el municipio de Balboa. Existen otras actividades más puntuales, pero, a nuestro juicio, carecen de relevancia tanto numérica como económica.

Finalmente, no debemos olvidar, aunque su análisis no constituye un objetivo de nuestro trabajo, que el mayor recurso económico hoy día se deriva de las pensiones por jubilación de la Seguridad Social Agraria, lo cual es lógico en un área con un envejecimiento tan fuerte de la población, pero que, al mismo tiempo, nos da una idea del nivel de desarticulación economica al que se ha llegado.

\section{ALTERNATIVAS AL PROCESO DE DESARTICULACIÓN}

En los numerosos artículos periodísticos y en algunos estudios realizados sobre esta comarca existe cierta tendencia a describir el proceso de desertización y abandono al que se está llegando, sin entrar a analizar las posibles alternativas -si es que existen- para frenar este proceso; alternativas que no deben plantearse de espaldas a las gentes que viven en la zona, pues, a nuestro juicio, no se trata de volver a períodos de «superpoblación», sino de crear las condiciones económicas que hagan atractiva la permanencia en estos municipios de personas jóvenes, aprovechando los recursos económicos y naturales que ofrece la zona y sin que por ello, su posible nivel de vida se constituya en un factor de expulsión de esta población.

En los últimos años hemos asistido a la realización de obras de infraestructura por parte de las diversas administraciones en base a la creacion de $\mathrm{Co}$ marcas de Acción Especial. Es indudable que estas intervenciones han contribuido a elevar el nivel de vida de la comarca, aunque el punto de partida era tan bajo que, en muchos casos, no se ha logrado superar aún cierta sensación de «espacios claustrofóbicos».

Sin embargo, lo que no han logrado esas intervenciones es propiciar un desarrollo económico que contribuya a frenar el proceso de desarticulación del que hemos hablado; simplemente han contribuido a mitigar ese proceso crítico e irreversible conducente a la despoblación casi total de la zona.

Observamos que no hay una plasmación ni de ayudas concretas ni de planes integrales que empiecen a introducir cambios apreciables o significativos es estos espacios. Uno de los objetivos que nos hemos marcado en nuestro trabajo de campo ha sido intercambiar opiniones con los residentes en la comarca; la conclusion más inmediata a la que llegamos es que hay una corriente de desánimo, cuando no de fatalismo, entre la mayoría de la población. Las excepciones vienen dadas por algunas personas jovenes retornadas de la emigración o que se han quedado en los pueblos por variadas circunstancias y que intentan conseguir ayudas económicas para diversas activida- 
des.

Es más, en la actualidad, un factor que contribuye a acentuar más el proceso de desarticulación es la «reconversión minera» que afectará a empleos directos de personas que, bien desplazándose diariamente, bien residiendo en algunas de las villas proximas (Fabero, Vega de Espinareda o Villäfranca -mina de Rubiais-), mantienen relaciones con sus lugares de origen en períodos de fin de semana o de vacaciones. Por otra parte, no queremos pensar en la incidencia que este proceso puede tener en el municipio de Peranzanes.

El problema fundamental es que el modo de producción vigente no encuentra factores de explotación que permitan una inversión de capital foráneo que integre estos espacios dentro de los flujos de desarrollo como ha sucedido en otros como las cuencas mineras, las canteras de pizarra o incluso rutas turísticas y vías de comunicación de primer orden que contribuyan al asentamiento de emigrantes retornados o, cuando menos, a una paralización de la emigración.

Debido al interés que esta comarca ha despertado a nivel provincial e incluso nacional, ya hemos señalado que son relativamente abundantes los trabajos, sobre todo periodísticos, algunos de los cuales intentan aportar soIuciones.

Por nuestra parte, consideramos que lo dificil es encontrar soluciones o alternativas que sean viables. No creemos que exista una actividad concreta cuya potenciación pueda ser el motor de desarrollo económico que no sólo frene el proceso al que nos hemos referido, sino que, al mismo tiempo, en torno a ella se articule económicamente la zona. Las posibles alternativas tienen que venir por la conjunción de diversas actividades de tipo tradicional, pero reorientadas, con la explotación de las potencialidades que ofrece la zona en nuevas actividades. Pero, para ello, partimos del pleno convencimiento de que la única salida, en un primer momento, sería una actuación de intensidad indiscutible por parte de las distintas administraciones, a través de los Ayuntamientos, para crear las bases que permitan el desarrollo de esas actividades. Dicho de otra forma, el nivel de desarticulacion demográfica y economica al que se ha llegado es tal que difícilmente se vislumbran alternativas «desde dentro» que, por otra parte, ya se hubiesen producido. Cuando hablamos de actuación por parte de las administraciones no nos referimos solamente al aspecto económico, sino también a la información y a la toma de iniciativas y de decisiones concretas que puedan fomentar el desarrollo de las actividades a las que nos vamos a referir.

Creemos que las actividades cuyo desarrollo podría servir de motor para la articulación económica de la comarca son: la potenciación de los recursos ganaderos, el aprovechamiento y puesta en explotación de los recursos forestales y los recursos turísticos; junto a ellas existen otras que pueden ser perfectamente complementarias.

La potenciación de los recursos ganaderos. Ya hemos señalado las aptitudes naturales de la zona para la explotación ganadera de distintas especies, pero, fundamentalmente bovino, ovino y caprino a pesar de que histórica- 
mente se haya dedicado un mayor esfuerzo a las labores agrícolas,

Recientemente Celada y Llamazares $(1989 ; 28)$ han Hegado a la conclusion, para las comarcas de Balboa y Somoza, de que «el territorio que nos ocupa es una zona especialmente apta para el desarrollo de una explotación animal extensiva, constituyendo ésta probablemente la única forma razonable de aprovechamiento, más si se tiene en cuenta que la vocación tradicional de esta tierra es el pastoreo». Esta apreciación se puede ampliar perfectamente a los valles vecinos de Ancares y Fornela.

Esta potenciación que propugnamos choca, a nuestro juicio, con una serie de dificultades sobre las que sería necesario actuar. Consideramos en primer lugar que el tema de la propiedad de la tierra en el espacio que estamos estudiando tiene una importancia decisiva en el sentido de que la conciencia de propietarios permanece extremadamente acusada por razones históricas como ya hemos demostrado en algún trabajo anterior (GONZÁLEZ VEcín, 1984). Constituyó un gran sacrificio por parte de los antepasados de los actuales labradores-ganaderos el acceso a la propiedad de la tierra en toda la montaña noroccidental berciana, de tal forma que todavía sigue presente en la mentalidad actual, manteniendo en gran parte estancado el mercado de la tierra y perdurando un régimen de propiedad totalmente anacrónico por el minifundismo y miniparcelismo existente. Incluso ciertas formas de arrendamiento y ciertos usos del suelo son una consecuencia de lo señalado anteriormente. La causa de todo esto es el ansia histórica por poseer la tierra que se trabaja y el costoso acceso a ella ${ }^{15}$.

En segundo lugar, como ya señalábamos al tratar el tema del aprovechamiento y uso del suelo, el terrero improductivo se ha visto incrementado en una apreciable cantidad de Has. Como consecuencia de la despoblación. Este terreno en un primer momento se aprovecha con fines ganaderos, pero posteriormente, pasa a incrementar la pérdida de pastos y pastizales por un aprovechamiento poco racional de los mismos.

Se debe fomentar también la utilización de pastos tipo braña aprovechando las estaciones climatológicamente más suaves para ganadería vacuno caballar y otras especies de ganado menor.

Creemos que se debe fomentar la introducción de plantas forrajeras (alfalfa, maíz, etc.) para ocupar aquellas tierras que ofrezcan posibilidades y que paulatinamente son abandonadas para usos agrícolas.

La Orientación de esta ganadería, en todas sus especies, sería fundamentalmente y prioritariamente hacia la producción de carne teniendo en cuenta

\footnotetext{
${ }^{15}$ En este sentido resulta interesante la consulta de los Repartimientos y Amillaramientos de los municipios estudiados (Archivo Histórico Provincial de León). Así en el municipio de Balboa en el año 1930 de 167 contribuyentes, aparecen 21 nuevos; en Peranzanes en el Apéndice al Amillaramiento de 1919 aparcen 45 altas, siendo la gran mayoría por compras y en 1955 de 29 altas, 11 son nuevos contribuyentes. En el mismo año en Paradaseca de 14 altas sólo dos son por herencia; las demás por compras. En el de 1920 ocurre algo parecido. Finalmente, en Candín de 334 contribuyentes en el Apéndice de 1927, 84 figuran como nuevos. Estos datos constituyen simplemente un ejemplo del trasiego de propiedades a lo largo de este siglo, al menos hasta los años sesenta.
} 
no tanto la cantidad -aspecto en el que difícilmente se podría competir con otro tipo de explotaciones- como la calidad basada en una alimentación que conjugue los aspectos tradicionales con innovaciones que son necesarias.

Creemos que de haberse fomentado este cambio en la orientación se hubiese evitado un proceso de despoblacion y abandono tan acusado.

Secundariamente podrían existir otros aprovechamientos ganaderos como el aprovechamiento de pieles o la elaboración de derivados lácteos de cara al mercado comarcal del Bierzo.

Aprovechamiento y puesta en explotación de Recursos Forestales. En absoluto entra en contradicción con la potenciación de los recursos ganaderos, pues las posibilidades que ofrece esta zona en cuanto a su aprovechamiento forestal son enormes. Sólo hay que tener en cuenta el porcentaje de terreno que ocupan los Montes de Utilidad Pública. Este tipo de recursos, en la actualidad, no s6́lo se caracteriza por su sinfraaprovechamiento» sino por su práctica inexistencia desde un punto de vista global. A nuestro juicio, debe partirse de repoblaciones de reposición con especies autoctonas (roble) o de nueva instalación con especies de crecimiento más rápido (fundamentalmente, pino) en las que la participación por parte de la población de la zona, tanto en el mantenimiento como en el consiguiente aprovechamiento económico debe ser algo fundamental.

También sería interesante la repoblación de especies de recolección (fundamentalmente castaño y nogal) con asesoramiento en cuanto a la comercialización de estos productos y buscando, sobre todo, la calidad de los mismos. Es evidente que las instituciones eluden cualquier responsabilidad a la hora de fomentar y crear, en sustitución de la iniciativa privada -en muchos casos inexistente por la edad y falta de preparacion del vecindario-, empresas dedicadas a la recepción y comercialización de estos productos que a largo plazo y a medida que esa experiencia se fuese adquiriendo, terminarían por convertirse en cooperativas o sociedades autogestionadas. También resulta evidente la falta de información sobre medidas tendentes a mejorar la cantidad y calidad de los productos.

El gran problema con el que ha de enfrentarse cualquier proyecto de repoblación es el de los incendios forestales; para tratar de evitarlos debe de ser implicada la población de la comarca mediante una información adecuada y haciéndola partícipe de los beneficios económicos que se deriven tanto en la creación de puestos de trabajo a la hora de repoblar como, cuando a más largo plazo, se produzca la tala de árboles. Debe ser facilitada, con el control necesario, la quema de rastrojos o maleza para evitar que cierto «furtivismo» produzca males mayores y siempre debe existir una vigilancia y el consiguiente castigo para aquellos que, a pesar de todo lo anterior, consideren que, en un beneficio propio mal entendido, deben quemar el monte. En este sentido habrá que esperar los resultados de los acuerdos alcanzados en este año por la Mancomunidad de Municipios Bierzo-Oeste con las distintas administraciones provinciales y regionales sobre la prevencion de incendios. En esta Mancomunidad se encuentra el Municipio de Balboa y de obtenerse resultados positivos podría ser el camino a seguir. 
Aprovechamiento y puesta en explotación de los recursos turísticos. La frecuente aparición de la Comarca de los Ancares en los medios de comunicación e incluso en trabajos de planificación del espacio rural, han descubierto para amplios sectores de poblacion urbana de la provincia y de fuera de ella un espacio en el que tanto los paisajes naturales como la pervivencia de modos de vida tradicionales llaman poderosamente la atención y constituyen un motivo de interés creciente ${ }^{16}$. Todo ello ha llevado a pensar en esta actividad -el llamado turismo rural, turismo verde, agroturismo...etc. -como en la panacea capaz de solucionar todos los problemas que aquejan a esta zona sin tener en cuenta, en muchas ocasiones, las características de este potencial turismo: turismo no masivo, to que constituye uno de sus atractivos; normalmente de corta duración y con un carácter «cultural» en un sentido amplio que abarcaría desde el disfrute de la naturaleza al conocimiento de la cultura tradicional de la zona.

Ello nos lleva a pensar en el turismo como uno de los recursos, -pero uno más- a tener en cuenta. Lo que sí está claro es que existe una urgente necesidad de planificar el desarrollo turístico de estas comarcas y para ello se deben llevar a cabo una serie de acciones tendentes a la ordenación de los recursos de este tipo.

Entre otras, destacamos las siguientes:

- Medidas tendentes a preservar el medio natural: en primer lugar se encuentra el tema de los incendios forestales al que ya hemos hecho referencia, pero también existen otras propias de la gestión municipal como la recogida de basuras (los ríos se han convertido en los basureros de la zona) que, afortunadamente, en alguno de los municipios estudiados ya es un hecho, aunque desde hace escasamente unos meses; o el tratar de evitar la invasión de actividades tales como competiciones de vehículos todoterreno que, a veces se promueven con un afán de promoción turística mal entendida desde las propias instituciones municipales.

- Medidas tendentes a la promoción de la zona: los recursos deben ser objeto de una gestión y asesoramiento técnico con el establecimiento de rutas trazadas por especialistas que permitan un conocimiento más completo y exhaustivo de estas comarcas. Debe existir la posibilidad de información sobre aspectos relacionados con la flora, la fauna, aspectos geológicos y geomorfológicos, modos de vida, recursos etnologicos, artísticos e históricos.

- Medidas tendentes al mantenimiento de los recursos: se deben crear «museos etnológicos» que permitan la conservación del riquísimo utillaje agrtcola y ganadero que actualmente se encuentra en vías de desaparición. En los últimos años se ha producido una renovación casi total del hábitat que incluso puede contradecir la dinámica que nosotros hemos descrito, aunque

\footnotetext{
${ }^{16}$ En este sentido destaca la creación por la Diputación Provincial de León de dos Oficinas de turismo, una en Villafranca del Bierzo y otra en Candín, que han comenzado a funcionar este verano.
} 
la explicacion se encuentra en diversos factores como la «intencion de pervivencia» por parte de jubilados o la creación de un hábitat estacional (a veces sólo el mes de agosto) para ocuparlo en vacaciones por personas originarias de esta zona. Pues bien, esta renovación total ha presentado como contrapartida la destrucción y casi desaparición del hábitat tradicional, pallozas y hórreos fundamentalmente. La política llevada a cabo en los años setenta en este sentido ha sido funesta, pero todavía en la actualidad se producen inversiones costosas contra las que no tenemos nada, pero, al mismo tiempo, no se tienden a mantener las pocas pallozas existentes o, al menos, no se ofrecen las ayudas que permitan mantener esta riqueza cultural autóctona con niveles y calidad de vida propios del siglo XX. La consecuencia es clara: el poblado de Campo del Agua ha sido pasto de las llamas, desgraciadamente, mientras que pallozas existentes en el centro de los núcleos de población como ocurre en Cantejeira (municipio de Balboa) o en el mismo Aira da Pedra (municipio de Balboa), en Peranzanes o en Burbia, se caen poco a poco porque su «teito» no es renovado y consiguientemente se pudren las maderas. Son simplemente ejemplos que se podrían poner en cada uno de los municipios analizados, y debemos tener en cuenta que no se trata de obligar a nadie a vivir en una palloza; se trata al menos de mantener la fisonomía externa y la distribución tradicional, pero con interiores adecuados a las necesidades actuales. Proponemos que la Comisión de Patrimonio de Ia Junta de Castilla y León conjuntamente con la Xunta de Galicia declare de interés cultural el mantenimiento de este valioso -y actualmente escaso- legado cultural arquitectónico, apoyando económicamente y, por tanto, exigiendo, a los propietarios de los mismos su mantenimiento.

Finalmente, debe intentarse que los recursos económicos proporcionados por estas actividades reviertan en los habitantes de la zona, mediante el establecimiento de una red de alojamientos en casas de labranza, la creacion de algún Camping, ya que salvo el de Burbia que ha empezado a funcionar este mismo año, no existe ningún otro, por inconcebible que parezca...etc.

Otros recursos económicos que con frecuencia se mencionan tendrían un carácter complementario; en este sentido, destacaríamos la apicultura, en grave riesgo de desaparición, la cunicultura y la rica tradición chacinera. Estamos seguros de que los consumidores de artículos tan conocidos como el botillo, ciertos productos de huerta como la patata, o la miel que pueden adquirirse en los mercados urbanos, valorarían la diferencia de calidad entre los métodos industriales y los artesanales y, seguramente, el consiguiente mayor precio no sería un grave inconveniente para su desarrollo e implantación.

Probablemente en un futuro muy cercano, si nos atenemos a lo que está sucediendo en municipios muy próximos (Vega de Valcarce y Trabadelo), comiencen a ser explotadas canteras de piedra que con la excusa de crear unos puestos de trabajo precarios proporcionan pingües beneficios al que las explota y causa un daño irreparable en el medio natural al menos tal como se están explotando en este momento.

Creemos que estas propuestas pueden frenar el proceso de desarticulación 
de las bases económicas tradicionales al que nos hemos referido en la primera parte deI trabajo y constituirse en alternativa viable, sustituyendo ese sistema económico tradicional, y al mismo tiempo, logrando fijar un número relativamente importante de población joven, evitando así la proxima desaparición de numerosas entidades de población.

\section{FUENTES}

Catastro del Marqués de Ensenada. Archivo Historico Provincial. León. Entidades de Balboa, Cantejeira, Balouta, Candín, Paradaseca y Peranzanes.

Catastro de Rústica. Características Generales y Listado Alfabético. Libros de Cédulas de 1983 y 1988. Catastro de Rústica. León.

Censos Agrarios de 1962, 1972 y 1982.

Censos Generales Ganaderos de 1945, 1982 y 1986.

Censos de Población. Años 1900, 1910, 1920, 1930, 1940, 1950, 1960, 1970 y 1981. I.NE.

MADOZ, P.: Diccionario geografico estadístico-histórico de España y sus posesiones de ultramar. Imprenta de D. José Rojas. Madrid 1845-1850.

Padron Municipal de Habitantes. Año 1975 y 1986. I.N.E.

Repartimientos, Amillaramientos y Apéndices. Archivo Histórico Provincial.

León. (Para los cuatro municipios).

Sección de Estadística e Informática del Servicio Territorial de la Junta de Castilla y León. León. Datos del año 1988 relativos a ganadería.

\section{BIBLIOGRAFÍA}

CELADA, J. D. y LLAMAZARES, T. E. (1989) Un estudio de ganadería extensiva de montaña: antecedentes históricos, estado actual y bases para su desarrollo en las comarcas bercianas de Somoza y Balboa. Leon. Mecanografiado.

GONZÁLEZ VECÍN, J. (1984) Geografía Social y Económica del Bierzo. Madrid: Servicio de Reprografía Universidad Complutense.

GONZÁLEZ VECÍN, J. (dir.) Análisis crítico de un proceso irreversible: la desarticulación de los Ancares. (Mecanografiado).

OTERO PEDRAYO, R. (1926) Guta de Galicia. Madrid: Espasa Calpe. TERÁN ÁLVAREZ, M. de (1952-56) Geografía de España y Portugal. Barcelona: Montaner y Simón.

RESUMEN: En el presente estudio se pretende analizar la evolución espectacularmente negativa de la población, que ha pasado en pocas décadas de una situación de superpoblación, a otra de despoblación y grave envejecimiento, sin perspectivas de relevo generacional.

La desarticulación de la economía y el modo de producción tradicional, sin que haya sido sustituido por otro nuevo, perviviendio aquél, pero totalmente degradado.

Uno de los factores que han contribuido y contribuyen a mantener la degradación, la desarticulación y la despoblación de los Ancares, es sin duda el régimen de tenencia de la tierra y los usos del suelo, que perduran como un anacronismo y contribuyen a bloquear posibles 
alternativas para transformar la comarca.

Algunas de estas alternativas agrícola-ganaderas, artesanales y turísticas constituyen la propuesta final de este trabajo. Sustentadas en el análisis científico de las estadísticas, elaboradas a partir de las distintas fuentes consultadas y de las encuestas y entrevistas realizadas en el trabajo de campo.

PALABRAS CLAVE: Minifundio, Catastro de Rústuca, erial, policultivo, agricultura de autoconsumo, envejecimiento demográfico, amiliaramientos.

RÉSUMÉ: Dans cette étude nous voulons analyser l'évolution spectaculairement négative, qui en quelques décennies est passé d'une situation de surpeuplement à une autre de dépeuplement et d'un important vieillissement, sans aucune perspective possible de remplacement.

La désarticulation de l'économie et le mode de production traditionel, sans avoir été remplacé par un autre plus moderne, en continuant à utiliser làutre, maís totalement hors d'usage. Un des facteurs qui ont contribué et qui contribuent encore à maintenir la dégradation, la désarticulation et le dépeuplement dans la region des Ancares, est sans doute le régime de possession de la terre et les usages du sol, qui perdurent comme un anachronisme et contribuent à bloquer des alternatives possibles afin de transformer la région.

Quelques unes de ces alternatives agricoles et d'élevage, artisanales et touristiques constituent le bout final du présent article. Ces propositions sont nourries d'une analyse scientifique des statistiques, élaborées à partir des différentes sources d'information consultées et des enquêtes et interviews réalisées sur le travail dans le champs.

MOTS CLÊ: Petite propriété, Cadastre Rustique, terres en friche, polyculture, agriculture d'autoconsomation, vieillissement démographique, cadastres.

SUMMARY: This study is aimed at analysing the notably negative evolution of the population, which in few decades has gone from a situation of overpopulation to one of depopulation and serious aging of the existing population with no prospects of generational replacement.

Also analysed is the disarticulation of the economy and the traditional mode of production which has not been replaced by another, but continues as before, though completely degraded. One of the factors that has contributed and still contributes to maintain the progressive diminution, the disarticulation and the depopulation of Los Ancares, is without doubt the system of tenure of the land and the way in which it is used, which still continue, although anachronistic, and contribute to bolcking possible alternatives that could transform the region.

Some possible alternatives in farming and livestock, artisanry and tourism are suggested at the end of this paper. They are based on scientific analysis of the statistics which have been compiled from the different sources consulted and from research and interviews carried out «in situ».

KEY WORDS: Small farmstead, Rustic Cadastre, untilled land, diversified farming, selfsufficiency farming, demographic aging, tax assessment. 